\title{
Regulation of JAK/STAT signal pathway by miR-21 in the pathogenesis of juvenile idiopathic arthritis
}

\author{
Hong-Wei $\mathrm{Li}^{1,2} \cdot$ Hua-Song Zeng ${ }^{1,2}$
}

Received: 27 January 2019 / Accepted: 7 May 2019 / Published online: 22 October 2019

(c) The Author(s) 2019

\begin{abstract}
Background Overexpression of the components of the Janus kinase/signal transducer and activator of transcription (JAK/ STAT) signalling pathway is the key factor of the pathogenic mechanisms underlying systemic juvenile idiopathic arthritis (sJIA). The study aims to investigate the association between miR-21 and the JAK/STAT signal pathway in JIA.

Methods Total RNA was extracted from peripheral blood mononuclear cells (PBMCs) in active JIA patients. The relative expressions of miR-21, STAT3 and suppressor of cytokine signalling 3 in PBMCs were measured by real-time polymerase chain reaction and their expressions were measured by western blotting and dual-luciferase reported assay. Rheumatoid arthritis fibroblast-like synovial cell (RASF) was stimulated to become to osteoclasts using macrophage colony-stimulating factor (M-CSF) and factors that can impact on their differentiation ability were identified through the transfection of LV3miR-21. The expression of STAT3/p-STAT3 was measured by western blot, and the levels of interleukin (IL)-17A, p65, matrix metalloproteinases (MMP)-3, MMP-4 and receptor activator of nuclear factor- $\mathrm{kB}$ after the LV3-miR-21 transfection were tested by enzyme-linked immunosorbent assay. Finally, the miR-21 targeted STAT3 gene was detected by the dualluciferase reported assay.

Results The expression of miR-21 was significantly lower in JIA patients than in healthy control $(P<0.05)$. The level of STAT3 was increased in PBMCs of JIA group compared with control group $(P<0.05)$. Furthermore, the expression levels of miR-21 in sJIA and polyarticular JIA groups were negatively correlated with STAT3 $(r=-0.5854 / r=-0.6134, P<0.05)$. The expression of STAT3 changed little in PBMCS after the stimulation of IL-6 and not in RASFs with transfection of LV3miR-21. The expression of p-STAT3 decreased after the stimulation of IL-6 in RASFs transfected by LV3-miR-21 $(P<0.05)$. RASFs were induced into osteoclasts using M-CSF. The number of osteoclasts as determined by tartrate-resistant acid phosphatase staining was significantly lower in group miR-21 mimics as compared with the negative control group $(P<0.05)$.

Conclusions We showed that expression of miR-21 was significantly lower in JIA patients compared with healthy control. MiR-21 might affect the JAK/STAT signal pathway by suppressing the expression of STAT3 and phosphorylation of STAT3. MiR-21 could inhibit the production of osteoclasts induced from RASFs by M-CSF.
\end{abstract}

Keywords JAK/STAT $\cdot$ Juvenile idiopathic arthritis $\cdot$ MiR-21 $\cdot$ Signal transducer and activator of transcription 3 (STAT3)/ p-STAT3

\section{Introduction}

Hua-Song Zeng

huasongxuqing@163.com

1 Department of Pediatric Allergy Immunology and Rheumatology, Guangzhou Women and Children Medical Center, Guangzhou 510623, China

2 Department of Pediatric Allergy, Immunology and Rheumatology, Guangzhou Women and Children's Medical Center, Guangzhou Medical University, 9 Jinsui Road, Guangzhou 510175, China
Juvenile idiopathic arthritis (JIA) is a chronic inflammatory disease which is characterized by persistent joint inflammation that manifests as joint pain and swelling while limiting the range of motion in the joints. It can lead to the shortterm and long-term disability $[1,2]$. This disease often presents with various clinical signs. However, there is usually non-specific laboratory evidence of inflammation at diagnosis and its pathogenesis is still ambiguous [2]. Therefore, JIA is difficult to diagnose early and its prognosis may be 
dependent on many factors. The current diagnosis of JIA relies mainly on clinical and radiographic findings, and may not provide correct diagnosis during the early stage of this disease. Thus, more sensitive and specific biomarkers are needed for an earlier diagnosis so that earlier treatment may improve the prognosis of these patients.

MicroRNAs (miRNAs) are non-coding RNAs, regulating gene expression of targeted mRNAs, which are implicated in the pathogenesis of autoimmune diseases [2]. MiRNAs can inhibit target gene function by binding combining to the 3 '-untranslater region (3'-UTR) of the targeted genes involved in cell growth, proliferation, differentiation and the regulation of the cell cycle [3]. Altered expression of miRNAs has been described under various pathological conditions, including rheumatic and other autoimmune diseases [4]. With the help of microarray analysis and quantitative polymerase chain reaction (qPCR), more and more miRNAs were found to be related to arthritis such as miR-16, miR155 [5], miR-146a [6], miR-223 and miR-132 [7]. Mature miR-146a and primary miR-146a/b were highly expressed in synovial tissue [8], and may reduce the expression of interleukin receptor associated kinase 1 [9]. Expression of miR223 is increased in polyarthritis and has been correlated with matrix metalloproteinase-3 (MMP3) [10]. However, there are few studies about the target genes of miRNAs that might demonstrate their roles in immunological dysregulation and chronic inflammation. Also, there are few studies about miRNAs on different subtypes of JIA.

MiR-21 is a well-known microRNA which is involved in the development of a variety of diseases, such as cancer and inflammatory diseases [11]. MiR-21, also has an important role in Treg biology, promoting Th17 differentiation depending on signal transducers and activators of transcription 3 (STAT3) and is preferentially activated by interleukin-6 (IL-6) [12-14]. The level of miR-21 was significantly lower in rheumatoid arthritis patients and was accompanied by the increase of STAT3, as well as a decrease of STAT5/ pSTAT5 protein and forkhead box P3 mRNA levels [15]. Another study showed that the high level of miR-21 could regulate the factor B cell lymphoma 6 (Bcl6), and then activate the expression of STAT3 $[4,5]$. Zhou et al. found that knockout of miR-21 could inhibit the expression of STAT3 in vivo [6]. Moreover, there are also some studies suggesting that STAT3 could influence the expression of miR-21 [16]. From the above data, it seems that miR-21 and STAT3 have a close relationship. But whether miR-21 exerts its function via combining STAT3 directly and whether mutual regulation of miR-21 and STAT3 has an impact on the inflammatory factors downstream is still unclear. As JIA is an inflammatory disease, we may ask, can the expression of miR-21 have an influence on the Janus kinase/signal transducer and activator of transcription (JAK/STAT) signal pathway in this disorder? It is also important to ask, what is the role of miR-21 in JIA?
In our previous study [17], we have explored the relationship between miR-21 and JAK/STAT signal pathway in rheumatoid peripheral blood mononuclear cells (PBMCs). However, it still has not been identified if miR-21 can exert its function by combining STAT3 directly. In this study, the aim was to find the exact mechanism of miR-21 and STAT3 functioning in the chronic inflammation and osseous destruction of JIA using specific methods such as dual-luciferase reporter, mutant of STAT3 3'-UTR and western blot for detecting the downstream protein expression of STAT3.

\section{Methods}

\section{Baseline collection}

In our previous study [17], thirty-three active phase JIA patients, aged from 2 to 14 years, who were seen in the Rheumatology Department of Guangzhou Women and Children's Medical Center, May 2017-July 2018, were included in this study. They belonged to two subgroups of JIA: systemic juvenile idiopathic arthritis (sJIA) (data were reported in a previous publication) [17] and polyarticular juvenile idiopathic arthritis (pJIA), conforming to the JIA classification of the 2001 International League of Associations for Rheumatology [18]. Twenty normal control patients were recruited from the Health Care Section of the hospital (the control data were reported in the previous publication) [17]. In this study, the patients had not started to use steroid or disease-modifying anti-rheumatic drugs after diagnosis and patients with other chronic diseases such as infections, hematopathy, and tumors were excluded. All the sampling were take in fasting condition. The study was performed based on an informed consent and was approved by the Ethics Committee of Guangzhou Women and Children's Hospital, Guangzhou, Guangdong, China. Informed written consent was obtained from all individuals. The baseline characteristics are shown in Table 1.

\section{Reagents}

Ficoll-Hypaque PLUS solution and phosphate-buffered saline (PBS) were purchased from GE Healthcare Life Sciences (Logan, UT, USA); the RNA extraction kit (9112; Takara Biotechnology Co. Ltd. Dalian, China), reverse transcription kit, RNAiso (9753A), miR-X iRNA First Strand Synthesis kit (638515) and SYBR Premix EX Taq (Tli R NaseH Plus; RR420A) kits were purchased from Takara Biotechnology Co, Ltd. The cell culture medium and materials were OptiMEM medium (31985-062; Gibco, USA), DMEM (11965-092; Gbico, USA), fetal bovine serum (FBS) (1168944; Gibco, USA), Trypsin (Gibco, USA), penicillin-streptomycin (Pen/Strep) (15140-122; Gibco, USA). 
Table 1 Comparison of baseline characteristic between patients with juvenile idiopathic arthritis and healthy control

\begin{tabular}{llll}
\hline Clinical characteristics & \multicolumn{2}{l}{ Case group $(n=33)$} & Control group $(n=20)$ \\
\cline { 2 - 4 } & sJIA group $(n=20)$ & pJIA group $(n=13)$ & \\
\hline Age $(\mathrm{y})$, mean \pm SD & $7.36 \pm 2.71$ & $6.80 \pm 2.23$ & $6.56 \pm 2.18$ \\
Sex (male:female) & $13: 7$ & $8: 5$ & $14: 6$ \\
Weight $(\mathrm{kg})$, mean \pm SD & $13.45 \pm 6.34$ & $14.17 \pm 5.47$ & $12.67 \pm 7.83$ \\
DAS28, mean \pm SD & $6.23 \pm 2.34$ & $6.17 \pm 1.78$ & - \\
MRI (synovitis/erosion), $n(\%)$ & $16(80)$ & $13(100)$ & - \\
Anti-AKA positivity, $n(\%)$ & $1(5.0)$ & $7(53.3)$ & - \\
Anti-CCP positivity, $n(\%)$ & $2(10.0)$ & $10(76.9)$ & - \\
RF-IgG positivity, $n(\%)$ & $15(75.0)$ & $9(69.2)$ & - \\
FER (ng/mL), mean \pm SD & $1079.47 \pm 900.87$ & $138.12 \pm 45.14$ & - \\
CRP (mg/L), mean \pm SD & $98.61 \pm 63.80$ & $89.74 \pm 34.85$ & 1.073 \\
ESR (mm/h), mean \pm SD & $53.12 \pm 26.59$ & $37.00 \pm 19.32$ & $2.57 \pm 1.15$ \\
\hline
\end{tabular}

SJIA systemic juvenile idiopathic arthritis, $p J I A$ polyarticular juvenile idiopathic arthritis, $C C P$ cyclic citrullinated peptide, $A K A$ anti-keratin antibody, FER ferritin, $R F-\operatorname{Ig} G$ rheumatoid factor $\operatorname{IgG}, C R P$ C-reactive protein, ESR erythrocyte sedimentation rate, $M R I$ magnetic resonance imaging, $S D$ standard deviation

LV3-miR-21 (Gene Pharma, 293 T cell, COA-020 3.0 $\times 10^{9}$ / mL, TAGCTTATCAGACTGATGTTGA), LV3-negative control (NC) (Gene Pharma, 293 T cell, C22AZ $2.0 \times 10^{9}$ / mL, TTCTCCGAACGTGTCACGTTTC).

\section{Isolation of total RNA from PBMCs and CDNA synthesis}

$3 \mathrm{~mL}$ blood was collected from JIA patients and healthy volunteers, in sodium ethylene diamine tetraacetate anticoagulant tubes and diluted in. $4 \mathrm{~mL}$ of PBS equal volumes of Ficoll Paque PLUS solution and diluted blood were added into $15 \mathrm{~mL}$ centrifuge tubes and centrifuged at $160 \times g$ for 20 minutes at $18-21{ }^{\circ} \mathrm{C}$. The cell pellet was then collected, resuspended in PBS, and centrifuged at $160 \times g$ for 20 minutes for 2 cycles. $1 \mathrm{~mL}$ RNAiso and 200-300 mL chloroform (Sigma-Aldrich, St. Louis, MO, USA) was added to the cell pellet and the samples were incubated for 5 minutes at $25-28{ }^{\circ} \mathrm{C}$ and centrifuged at $12,000 \times g$ for 15 minutes at $4{ }^{\circ} \mathrm{C}$. Approximately $400 \mu \mathrm{L}$ supernatant was obtained, to which $1 \mathrm{~mL}$ cold isopropanol (Sigma-Aldrich) was added, mixed and incubated at $4{ }^{\circ} \mathrm{C}$ for 10 minutes, prior to being centrifuged at $12,000 \times g$ for 10 minutes at $4{ }^{\circ} \mathrm{C}$. The supernatant was then discarded, and $10 \mathrm{~mL} \mathrm{75 \%} \mathrm{ethanol} \mathrm{prepared} \mathrm{with} \mathrm{diethylpyrocarbonate}$ (DEPC) (Sigma-Aldrich) water was added (ethanol:DEPC $3: 1$ ), followed by centrifugation at $7500 \times g$ for 5 minutes at $4{ }^{\circ} \mathrm{C}$. Finally, $20 \mu \mathrm{L}$ DEPC water was added to the samples, and the absorbance was measured using a spectrophotometer (Thermo NanoDrop 2000; Thermo Fisher Scientifc, Inc, Waltham, MA, USA); reverse transcription of RNA into cDNA was done using miR-X iRNA First Strand Synthesis kit. cDNA were stored in $-20^{\circ} \mathrm{C}$. All manipulation was proceeded according to the manufacturer's protocol. Rheumatoid arthritis fibroblast-like synovial cells (RASFs) were from biopsy of JIA patients and stored in $-80^{\circ} \mathrm{C}$.

\section{Detecting the expression of miR-21 and its associated mRNAs in PBMCs by quantitative real-time PCR (qRT-PCR)}

The mRNA expression levels of specific genes were quantified by qRT-PCR. The primers of miR-21 (5'-GTCGTATCC AGTGCAGGGTCCGAGGTATTCGCACTGGATACGACT CAACA-3') and U6 (5'-CTCAACTGGTGTCGTGGAGTC GGCAATTCAGTTGAGAAAAATATG-3') were synthesized by TAKARA.

The primers of STAT3 (F: 5'-GCCAGAGAGCCAGGA GCA-3', R: 5'-ACACAGATAAACTTGGTCTTCAGG TATG-3'), suppressor of cytokine signaling 3 (SOCS3) (F: 5'-CAGCTCCAAGAGCGAGTACC-3', R: 5'-TGACGC TGAGCGTGAAGAAG-3'), $\beta$-actin (F: 5'-GAGCTACGA GCTGCCTGACG-3', R: 5'-GTAGTTTCGTGGATGCCA CAG-3') were synthesized by Beijing Genomics Institute.

Expression of MMP-3, MMP-4, receptor activator of nuclear factor- $\kappa \mathrm{B}$ ligand (RANKL) and NF-кb was detected after the LV3-miR-21 transfected RASFs were treated with IL-6 for 1, 2, 4 and 8 hours, respectively. The sequences of the primers were as follows:

MMP-3 (F: 5'-CACTCACAGACCTGACTCGGTT-3', R: 5'-AAGCAGGATCACAGTTGGCTGG-3'),

MMP-4 (F: 5'-CCTGACAAAGCACGGCAAGAAC-3', R: 5'-CCAGCACCTTGGAACTTCTGTC-3'),

RANKL (F: 5'-GCCTTTCAAGGAGCTGTGCAAAA-3', R: 5'-GAGCAAAAGGCTGAGCTTCAAGC-3'),

$N F-\kappa b$ (F: 5'-ACGATCTGTTTCCCCTCATC-3', R: 5'-GCTTCTCTCCCCAGGAATACTGCC-3'). 


\section{Cell culture and transfection of LV3-miR-21 into RASFs}

RASFs were thawed from storage and subcultured for $2-3$ times to $80-90 \%$ confluence. Cells were inoculated into 6 -well plates according to a cell density of $6 \times 10^{5}$ cells/ well. Serum-free medium was replaced the next day and multiplicity of infection (MOI) of 50 of virus was added to the culture, incubated for 2 hours and then culture media was replaced by fresh complete medium. After 48 hours, the cells were collected and passaged in 1:2. After adherence, the cells were screened by adding puromycin at a concentration of $10 \mu \mathrm{g} / \mathrm{mL}$. The solution was changed every 2-3 days (puromycin complete medium with $10 \mu \mathrm{g} / \mathrm{mL}$ ). One week later, the medium was replaced with puromycin complete medium of $2 \mu \mathrm{g} / \mathrm{mL}$. The RASFs were suspended in a concentration of $2 \times 10^{5}$ cells/mL in DMEM (11965-092, Gibco) supplemented with $10 \%$ heat-inactivated FBS and $1 \%$ Pen/ Strep in 6-well plates for 24 hours at $37^{\circ} \mathrm{C}$. When the cell density reached $70-80 \%$ confluence, the cells were transfected with LV3-miR-21 lentivirus vector (Gene Pharma) and LV3-NC according to the manufacturer's instructions. Cells were then harvested and subjected to qRT-PCR analysis of miR-21 expression.

\section{Cell viability assay}

RASFs were seeded into 96-well plates and incubated with treating with IL-6 of different concentrations $(0,1,10,100$, and $150 \mathrm{ng} / \mathrm{mL}$ ) for $0,0.5,1,2$, and 4 hours, respectively. Subsequently, $100 \mu \mathrm{L}$ fresh medium was added to cells containing $10 \mu \mathrm{L}$ Cell Counting Kit-8 solution (Dojindo Laboratories, Tokyo, Japan) and incubated for 1 hour $\left(37^{\circ} \mathrm{C}, 5 \%\right.$ $\mathrm{CO}_{2}$ ). Absorbance at $450 \mathrm{nM}$ was measured using a microplate reader (Cytation5, BioTech, USA).

\section{Inducing the differentiation of RASF-derived osteoclasts and identifying factors that can impact on their differential ability}

RASFs were inoculated on 6-well plates at a concentration of $1 \times 10^{5}$ cells per well. After culturing overnight, the medium was discarded and replaced with $2 \mathrm{~mL}$ of fresh medium. Macrophage colony-stimulating factor (M-CSF) was added to a final concentration of $30 \mathrm{ng} / \mathrm{mL}$. The cells were placed in a $37{ }^{\circ} \mathrm{C}, 5 \% \mathrm{CO}_{2}$ incubator for 5 days. The differentiation into osteoclasts was identified by tartrate-resistant acid phosphatase (TRAP) staining. Then the RASF-derived osteoclasts were divided into five groups: mock group, LVmiR-21 group, LV-miR-21 + phosphorylation inhibitors, LVmiR-21 + phosphorylation inhibitor mock and blank group.
To verify the differentiation ability of these groups, cells were treated with IL-6 $(100 \mathrm{ng} / \mathrm{mL})$ for 1, 2, 4 and 8 hours, respectively, and the cells were stained with TRAP.

\section{Dual-luciferase reported assay}

$293 \mathrm{~T}$ cells $\left(1 \times 10^{4}\right)$ were seeded in $500 \mu \mathrm{L}$ medium into wells of a 12-well plate. After 18-24 hours of incubation, cells were transfected for 1-2 hours with $1 \mu \mathrm{g}$ reporter plasmid (STAT3 3'-UTR and STAT3 Mut 3'-UTR) (Transgen, Beijing, China) using $250 \mu \mathrm{L}$ Optim and $3 \mu \mathrm{L}$ Lipofectamine 2000 according to the manufacturer's instructions. After transfection, the medium was replaced with $500 \mu \mathrm{L}$ medium with $10 \%$ FBS and incubated for another 6 hours. Dual-luciferase reporter assay was performed 48-72 hours after the transfection. Cells were harvested by adding $200 \mu \mathrm{L}$ passive lysis buffer at room temperature. After centrifuging, $100 \mu \mathrm{L}$ supernatant was assayed for luciferase detection using a microplate luminometer (Cytation5, BioTech, USA). Transcription factor-binding sites in promoter region of STAT3 with miR-21 was predicted with bioinformatics analysis.

\section{Western blot analysis}

RASFs $(n=5)$ were collected and dissolved using $200 \mu \mathrm{L}$ RIPA buffer (Beibo, China) with $2 \mathrm{mM}$ protease inhibitors sodium orthovanadate (Beibo, China) and $2 \mu \mathrm{L}$ phosphatase inhibitors (Beibo, China) for $30 \mathrm{~min}$ on ice and then centrifuged at $10,000 \mathrm{rpm}$ for 30 minutes at $4{ }^{\circ} \mathrm{C}$. After, the total protein concentrations were measured using the BCA method (Biyuntian, China). Equal amounts $(30 \mu \mathrm{g})$ of protein were separated by $10 \%$ sodium dodecyl sulfate denatured polyacrylamide gel and transferred onto poly (vinylidene fluoride) membranes in bovine serum albumin in Tris-based saline with Tween 20 for 1 hour at room temperature. Membranes were incubated with rabbit antihuman antibodies at the recommended dilution [STAT3 at a dilution of 1:100 (Abcam, USA), p-STAT3 (Tyr705) at 1:10,000 (Abcam, USA), SOCS3 at 1:10,000 (Abcam, USA), GAPDH at 1:5000 (Abcam, USA)] overnight at $4{ }^{\circ} \mathrm{C}$. After washing in Tris-based saline with Tween 20, the membranes were further incubated with a secondary anti-rabbit antibody $(1: 10,000)$ for 2 hours. Enhanced chemiluminescence solution was added onto the membranes, and protein expression was quantified using the Laboratory Work Image Acquisition and Analysis Software (UVP, Upland, CA). GAPDH was used as a loading control.

\section{Enzyme-linked immunosorbent assay (ELISA)}

Levels of IL-17A, p65, MMP-3, MMP-4 and RANKL were measured by ELISA according to the manufacturer's 

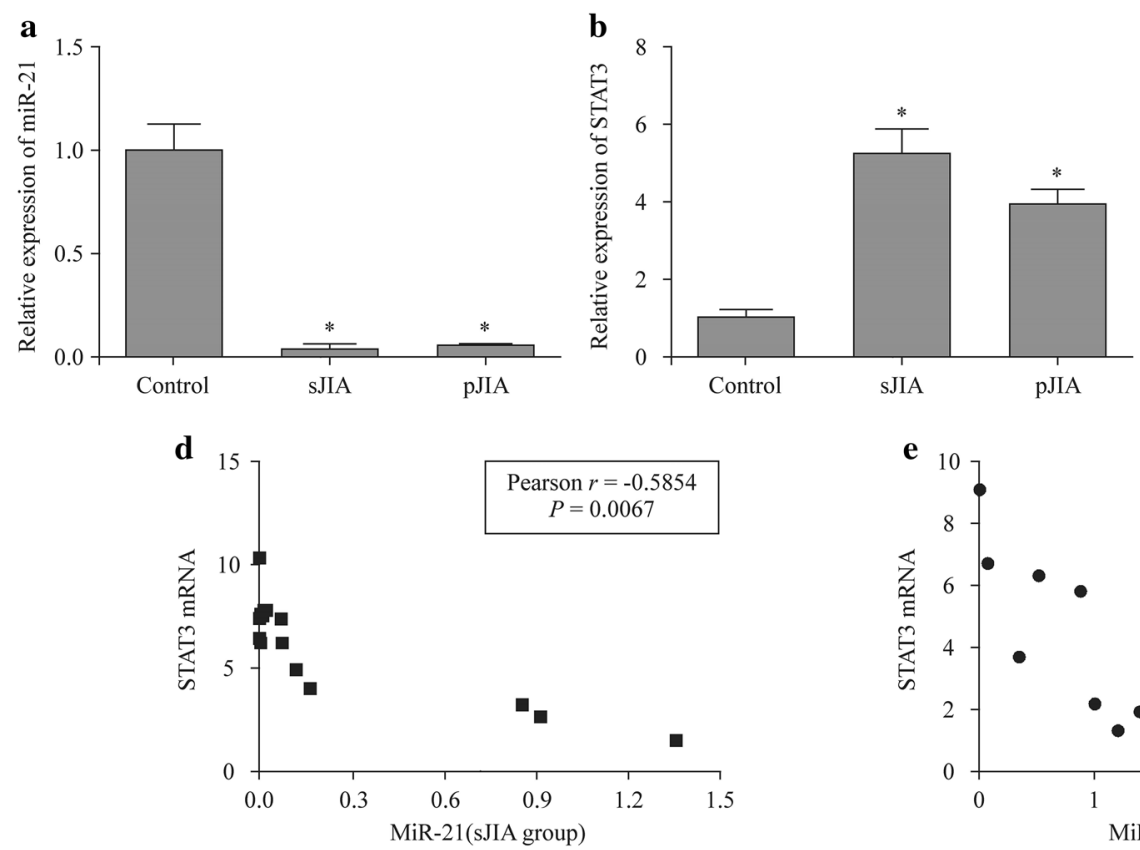

Fig. 1 Expression of miR-21, STAT3 and SOCS3 mRNA of PBMCs and their correlation in JIA. $s J I A$ systemic juvenile idiopathic arthritis, pJIA polyarticular juvenile idiopathic arthritis, STAT3 signal

protocols (USCN Life Science Inc., Wuhan, China; Kamiya Biomedical Co., KT-58997, Tukwila, WA, USA).

\section{Statistical analysis}

SPSS 11.0 software was used and all data were expressed as mean (SD). Differences between the two groups were assessed using Kruskal-Wallis test or Student's $t$ test, whereas the correlation of multiple groups was analyzed using Pearson's correlation coefficient. The Student's $t$ test was employed for pairwise comparisons between two groups in baseline, and two-way ANOVA was used for intergroup comparisons. $P<0.05$ indicated statistically significant.

\section{Results}

\section{Baseline}

For JIA [sJIA $(n=20)$, pJIA $(n=13)$ ] patients and healthy controls, their demographic and clinical characteristics were recorded, including age, sex, rheumatoid factors, anti-citrullinated protein antibody, C-reactive protein, erythrocyte sedimentation rate, magnetic resonance imaging and ferritin (FER) (Table 1). No significant difference was found in age, sex or weight between two groups $(P>0.05)$. There were significant differences in anti-keratin antibody (AKA),
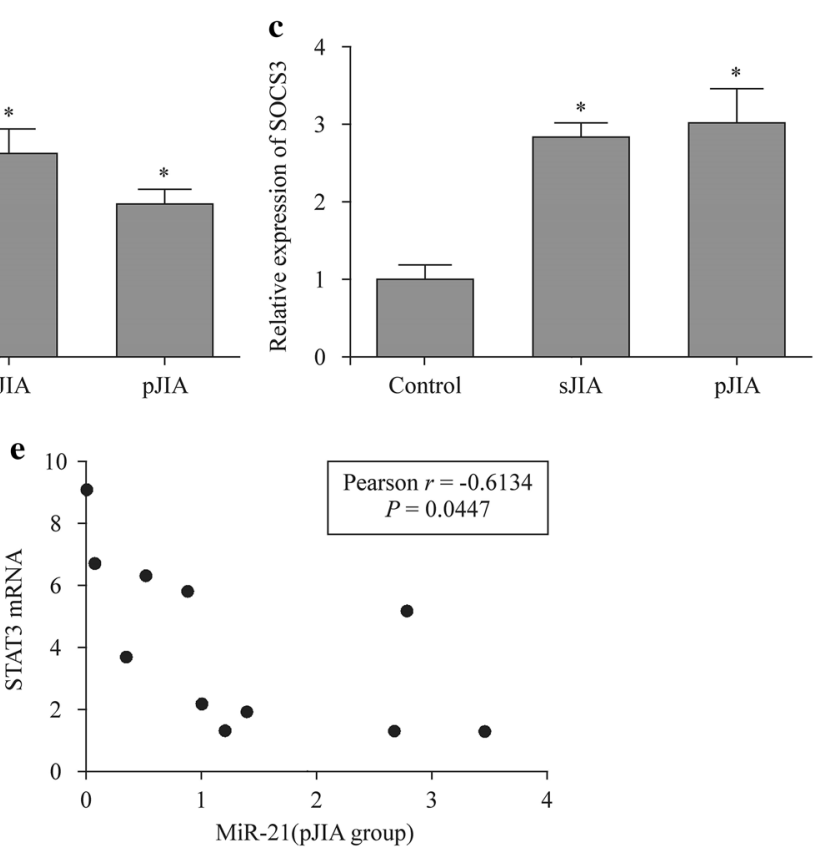

transducers and activators of transcription 3, SOCS3 suppressor of cytokine signaling $3, P B M C$ s peripheral blood mononuclear cells. * $P<0.05$

cyclic citrullinated peptide (CCP) and FER between the SJIA and pJIA groups $(P<0.05)$. Positive for RF IgG of sJIA was highly $75 \%(15 / 20)$ (Table 1$), 25 \%(5 / 20)$ positive in second review. The positive percentage of AKA and CCP was higher in the pJIA group compared with the sJIA group, while the mean level of FER increased in the sJIA group. The characteristics of baseline coincided with the study.

\section{Expression of miR-21, STAT3 and SOCS3 mRNA of PBMCs and their correlations in JIA}

In our previous study [19], we decided to compare the expression of miR-21 in PBMC and from JIA [sJIA $(n=20)$, pJIA $(n=13)]$ patients and healthy controls. Indeed, as shown in Fig. 1a, expression of miR-21 was significantly lower in JIA patients than the healthy controls $(P<0.05)$, and the miR-21 expression of group sJIA and group pJIA was 7.7 times and 6.49 times lower than the control groups (Table 2). However, there was no significant difference of miR-21 expression between group sJIA and pJIA. Moreover, level of STAT3, SOCS3, IL-6, tumor necrosis factor- $\alpha(T N F-\alpha)$ gene increased in PBMCs of JIA group compared with control group $(P<0.05)$ (Fig. 1b, c). We also found that the expression level of miR-21 in sJIA and pJIA group was correlated negatively with STAT3 $(r=-0.5854 / r=-0.6134, P<0.05)$ (Fig. 1d, e). 
Table 2 Expression of miR-21 and STAT3/SOCS3 in sJIA and pJIA group

\begin{tabular}{llllr}
\hline Group & \multicolumn{2}{l}{ Case group } & Control $(n=20)$ & Z/P value \\
\cline { 2 - 3 } & sJIA $(n=20)$ & pJIA $(n=13)$ & & \\
\hline MiR-21 & $7.70(7.00,8.50)$ & $6.49(6.00,7.00)$ & $1.02(0.64,1.45)$ & $2.615 / 0.015,2.334 / 0.029$ \\
STAT3 & $6.24(2.81,7.54)$ & $3.97(1.81,5.75)$ & $1.00(0.56,1.29)$ & $4.869 / 0.001,3.804 / 0.008$ \\
TNF- $\alpha$ & $3.03(2.07,3.80)$ & $3.42(2.46,4.68)$ & $1.09(0.93,2.01)$ & $3.356 / 0.002,5.146 / 0.001$ \\
IL-6 & $4.75(3.59,6.32)$ & $3.52(2.31,7.51)$ & $1.87(1.23,2.54)$ & $3.082 / 0.006,2.388 / 0.036$ \\
SOCS3 & $2.54(1.77,4.00)$ & $3.57(1.95,3.83)$ & $0.86(0.21,1.20)$ & $3.344 / 0.003,4.783 / 0.001$ \\
\hline
\end{tabular}

SJIA systemic juvenile idiopathic arthritis, $p J I A$ polyarticular juvenile idiopathic arthritis, STAT3 signal transducers and activators of transcription 3, SOCS3 suppressor of cytokine signaling 3,TNF- $\alpha$ tumor necrosis factor- $\alpha, I L-6$ interleukin-6

\section{Transfection of LV3-miR-21 suppressed p-STAT3/ STAT3 protein expression}

To investigate the connection between miR-21 and STAT3, LV3-miR-21 and LV3-NC were transformed into RASFs that could express p-STAT3/STAT3 proteins. After a 5-day transfection, the expression levels of miR-21 dramatically increased after LV3-miR-21 transfection (Fig. 2a, b). Then RASFs were stimulated with IL-6 $(0,10,100,150 \mu \mathrm{g} /$ $\mathrm{mL}$ ) for 4 hours (Fig. 2c). The upregulation of miR-21 in the RASFs was associated with suppression of phosphorylation of STAT3 and upregulation of SOCS3. However, level of STAT3 protein did not change by overexpressing miR-21 (Fig. 2d, e). Moreover, we found that LV3-miR-21 could suppress the protein translation of STAT3 in RASFs stimulated with IL-6 $(P<0.05)$, but no change had been found in the IL-6 absent group (Fig. $2 \mathrm{~d}-\mathrm{g}$ ). A typical presentation was shown that the STAT3 greatly increased with the presence of IL-6 in RASFs transfected with LV3-NC compared with control group while it decreased in LV3miR-21 group (Fig. 2h). While the p-STAT3 decreased in LV3-miR-21 group in absent of IL-6 and stimulation of IL-6 (Fig. 2i). The level of SOCS3 decreased in RASFs with the stimulation of IL-6 $(P<0.05)$ (Fig. $2 \mathrm{j})$.

\section{Enhancing miR-21 or silencing STAT3 could suppress the IL- 6 induced regulation of targeted genes}

RASF cells were divided into four groups: control, RASF + vector, RASF + LV-miR-21 and RASF + siRNASTAT3. They were treated with IL-6 $(100 \mathrm{ng} / \mathrm{mL})$ for 0,1 , 4, 8 hours. Then expression of $M M P-3, M M P-4, R A N K L$ and $N F-\kappa b$ was detected by qRT-PCR. It showed that the expression of these genes could be upregulated by treating IL-6, the optimal treating time was 4 hours (Fig. 3). However, increasing the expression of miR-21 with LV-miR-21 or silencing STAT3 with siRNA-STAT3 could effectively suppress this IL-6-induced effect (Fig. 3).

\section{Enhancing miR-21 or silencing STAT3 could suppress the IL-6-induced regulation of targeted proteins}

Four groups (control, RASF + vector, RASF + LV-miR-21 and RASF + siRNA-STAT3) of RASF cells were treated with IL-6 $(100 \mathrm{ng} / \mathrm{mL})$ for 4 hours, then the protein level of IL-17A (Fig. 4a), p65 (Fig. 4b), RANKL (Fig. 4c), MMP-3 (Fig. 4d) and MMP-4 (Fig. 4e) was measured by ELISA assays. The result showed that the levels of these proteins were significantly lower in groups of RASF + miR-21 and RSAF + siRNA-STAT3 (Fig. 4).

\section{MiR-21 exerts its functions via targeting STAT3 gene directly}

To further investigate whether miR-21 is a functional target of STAT3, a dual-luciferase reporter assay was performed. We predict and design hsa-miR-21-5p target 2040 to 2046 on STAT3 3'-UTR with bioinformatics analysis. The results showed that the luciferase activities of the cells with STAT33'-UTR dropped after being co-transfected with hsa-miR21-5p mimics, comparing with the control group using miR-21 mimic NC instead. The luciferase activity of the mimic group was $83.468 \%$ of the NC group, which meant that hsa-miR21-5p mimics could slightly inhibit the expression of gene STAT3. As a comparison, 293 T cells with mutant STAT3-3'UTR were also transfected with hsa-miR-21-5p mimics and miR-21 mimics NC. However, the decrease of luciferase activities of the mimic group was $97.523 \%$ of the NC group (Fig. 5).

\section{MiR-21 could inhibit the production of osteoclasts induced from RASFs by macrophage colony-stimulating factor (M-CSF)}

CSF was used for inducing the RASFs to develop into osteoclasts. Based on TRAP staining, the amounts of osteoclasts in group miR-21 mimics were significantly lower than the NC group $(P<0.05)$ (Fig. 6a). Moreover, the decrease of cell amounts could also be found in the NC group which 


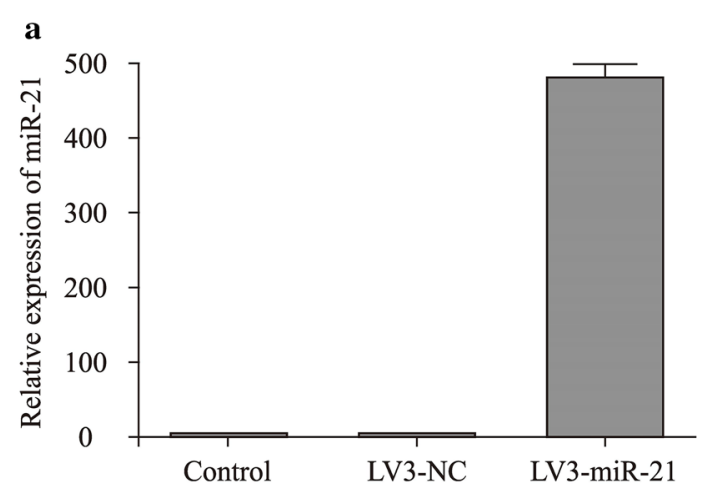

b
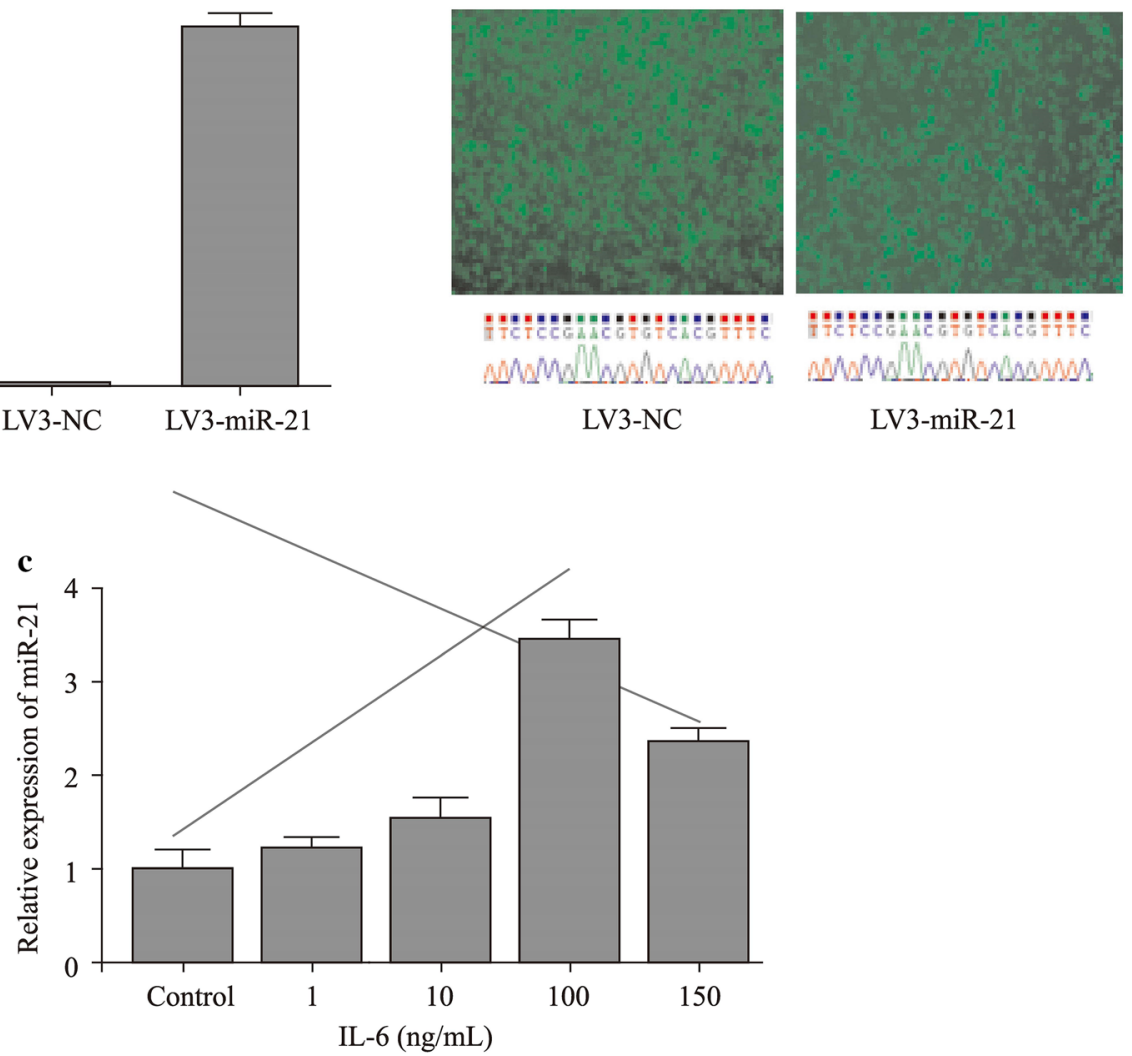

d

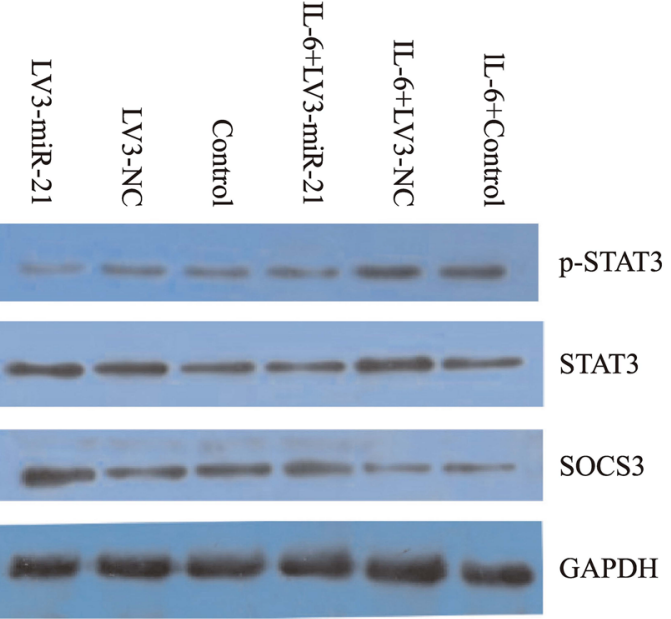

Fig. 2 Transfection of LV3-miR-21 suppressed p-STAT3/STAT3 protein expression: a, b The titer of miR-21 in RASFs after LV3-miR-21 transfection; $\mathbf{c}$ OD value of RASFs with the simulation of IL-6; $\mathbf{d}-\mathbf{j}$ The expression of STAT3/p-STAT3/SOCS3 in RASFs after transfection of LV3-miR-21 with the simulation of IL-6. STAT3 signal transducers and

was transfecting with miR-21 mimics after the treatment of inhibitor of STAT3 phosphorylation (cryptotanshinone) with no difference between the group miR-21 mimics and

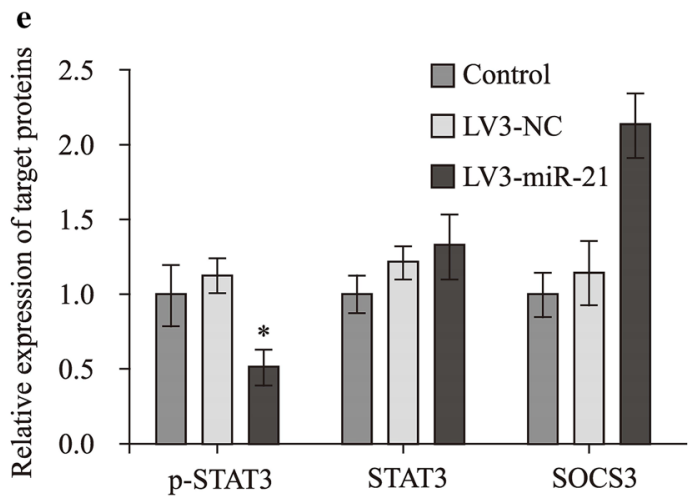

activators of transcription 3, SOCS3 suppressor of cytokine signaling 3, IL-6 interleukin-6, RASF rheumatoid arthritis fibroblast-like synovial cell, $N C$ negative control, $* P<0.05, \dagger P<0.05$

group STAT3 phosphorylation inhibition (Fig. 6b). Therefore, miR-21 has a similar function as STAT3 inhibitor on inhibiting the production of osteoclasts. 


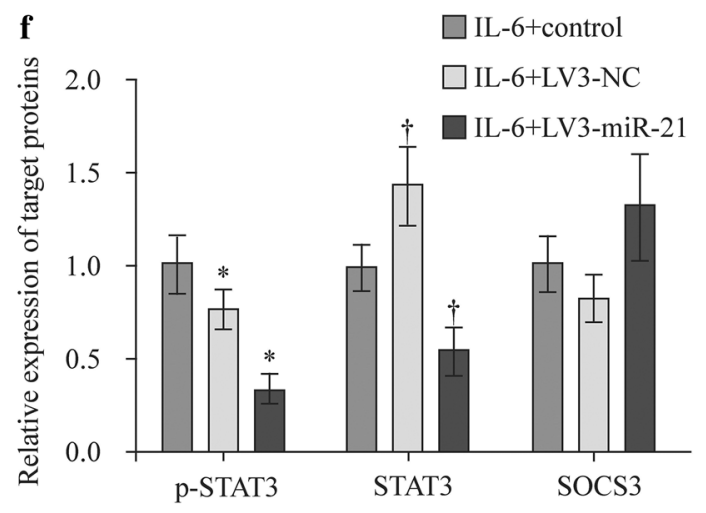

h

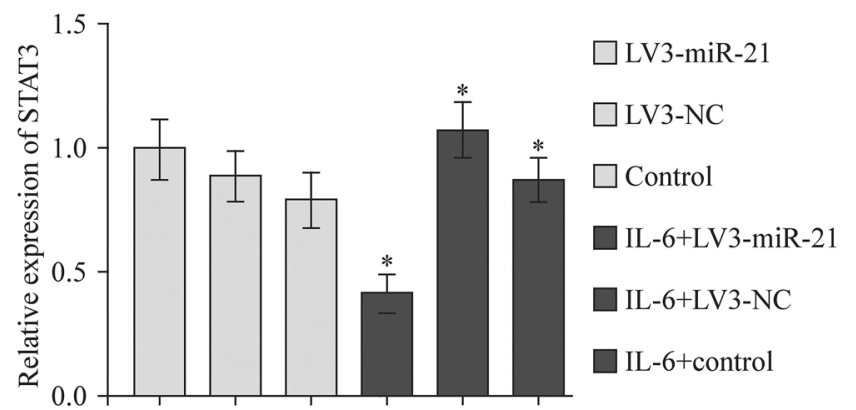

\section{j}

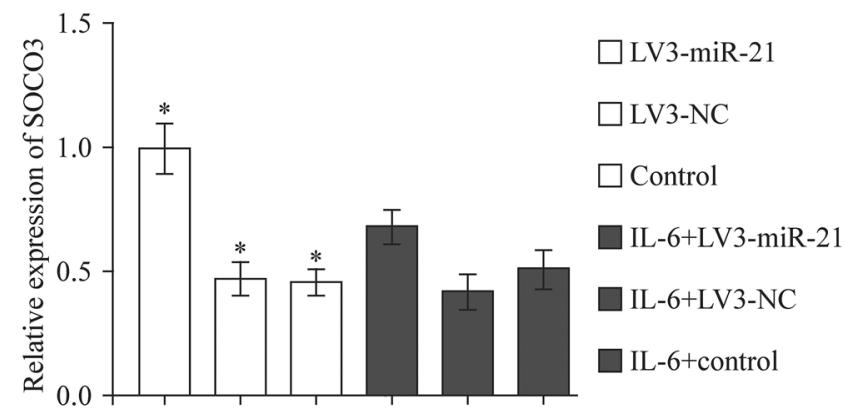

Fig. 2 (continued)

\section{Discussion}

JIA is a heterogeneous condition with a variable outcome and considerable ongoing disease burden [18]. MiR-21 has been described as an oncogenic miRNA exhibiting antiapoptotic activity in various carcinomas and inflammations. It can, not only regulate the level the inflammatory factor, such as TNF- $\alpha$ and IL-10 secretion [19], but also be induced by many pro-inflammatory stimuli, such as pathogen-associated molecular patterns and damage-associated molecular patterns. This suggests that miR-21 may be an important factor for the resolution of inflammation with the negative regulation of the inflammation process [20]. As shown in Fig. 1a, expression of miR-21 was significantly lower in JIA patients than healthy controls $(P<0.05)$. It may be helpful to
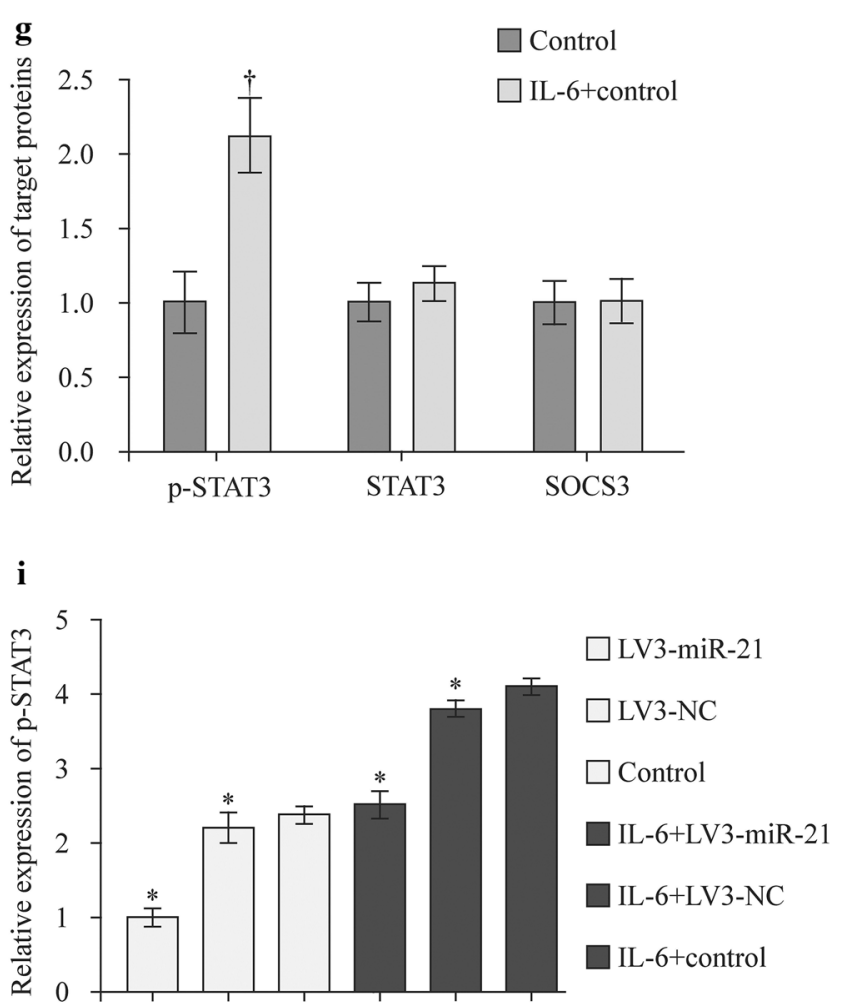

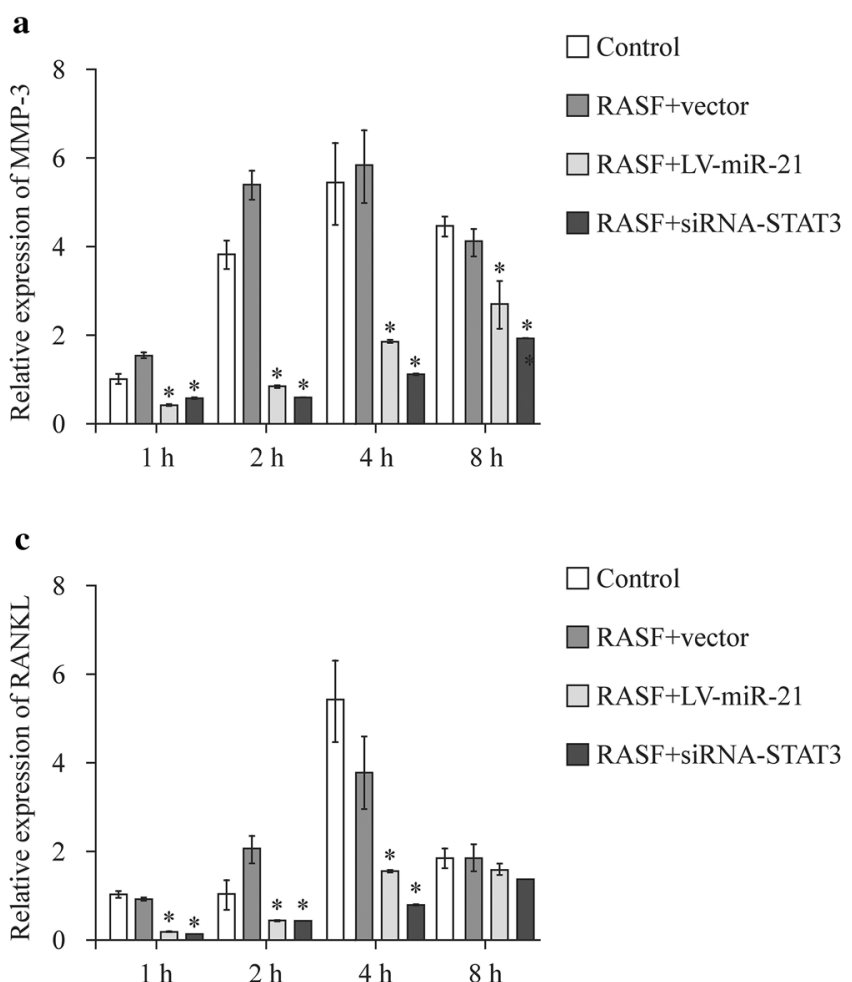

Fig. 3 Enhancing miR-21 or silencing STAT3 could suppress the IL-6 induced regulation of targeted genes. STAT3 signal transducers and activators of transcription 3, IL-6 interleukin-6, RASF rheuma-

cytokines, such as IL-6 $[25,26]$. After IL-6 stimulation, the target cell launches an intracellular signal transduction cascade via the JAK/STAT pathway, leading to the phosphorylation of STAT3 [27-29]. Indeed we had demonstrated that STAT3 were abnormally upregulated in the PBMCs of JIA patients as shown in Fig. 1b. Furthermore, we had found that the expression of miR-21 was negatively correlated with STAT3 (Fig. 1d, e). Therefore, whether STAT3 is the target gene for miR-21 is an important issue that needs resolution.

Our study showed that the expression level of miR21 was negatively correlated with STAT3 in PBMCs of JIA $(r=-0.5854 / r=-0.6134, P<0.05)$ (Fig. 1d, e). We inferred that STAT3 might suppress the expression of miR21 in PBMCs of JIA. But which role did miR-21 play in JAK/STAT signal pathway? Recent studies showed that STAT3 might correlate with IL-6-induced miR-21 [30]. Clinical data strongly indicated that IL-6 and IL-6R may play a key role in the induction and progression of JIA and its complications [31].

Data in Fig. 2h shows hardly any difference in STAT-3 whether IL-6 is added or not. The only difference is the addition of LV3-miR-21 inhibits STAT-3 expression in RASFs stimulated with IL-6, but LV3-miR21 transfection had no effect on STAT-3 expression when IL-6 is not added to the
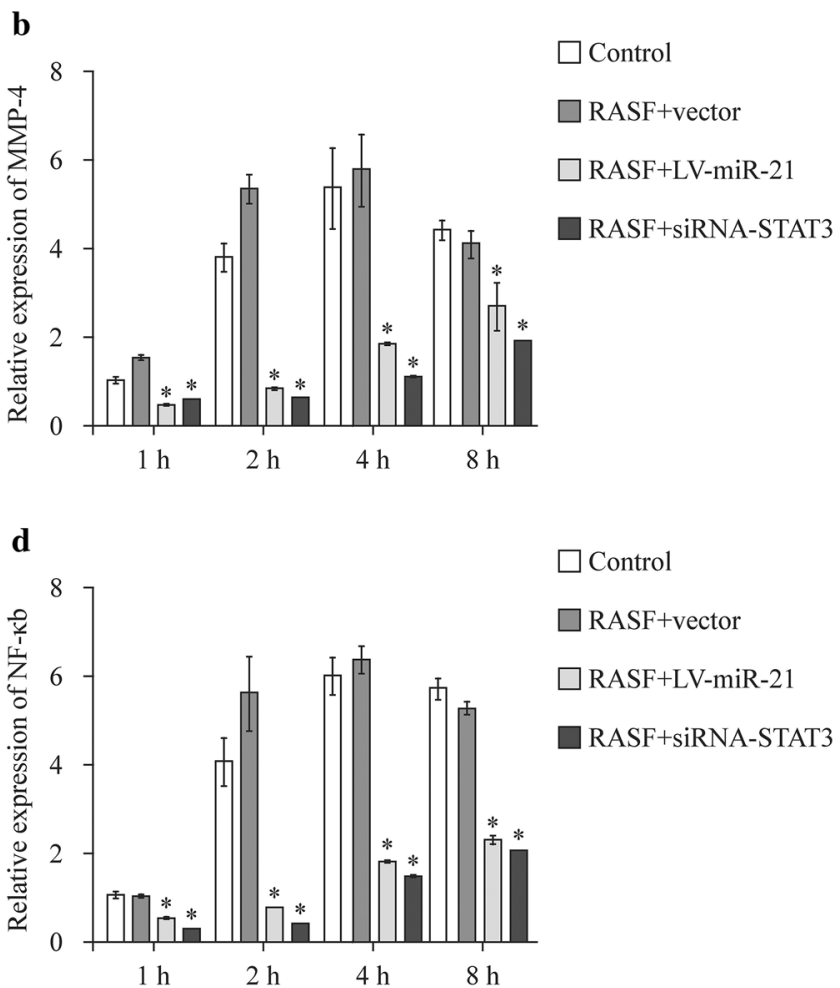

toid arthritis fibroblast-like synovial cell, $M M P$ matrix metalloproteinase, $R A N K L$ receptor activator of nuclear factor- $\kappa \mathrm{B}$ ligand, $N F-\kappa b$ nuclear factor- $\kappa \mathrm{B}$

culture system. So we inferred that over-expression of miR21 can decrease the level of STAT3 only during inflammation (as indicated by the presence of IL-6).

However, whether STAT3 is the target gene of miR-21 remains unknown. In our study, we showed that miR-21 could exert its functions by combining with the STAT3 gene directly by dual-luciferase assay. Hsa-miR-21-5p mimics (target by 2040 to 2046 on STAT3 3'-UTR) could slightly inhibit the expression of gene STAT3. As a comparison, 293 T cells with mutant STAT3-3'-UTR were also transfected with hsa-miR-21-5p mimics and miR-21 mimics NC. It can be concluded that gene miR-21 could inhibit proinflammatory activities and bone destruction by binding to its target gene STAT3.

Our findings showed that miR-21 could inhibit the phosphorylation of the target protein STAT3 (Fig. 2i). The p-STAT3 greatly increased with the presence of IL-6 in RASFs transfected with LV3-NC and in control group while decreased in LV3-miR-21 group, shown in Fig. 2i. A study showed the Bcl6 is an important regulator of Th cells, and could inhibit Th2 inflammation. At present, it is thought that miR-21 is the target of Bcl6, and is able to downregulate $\mathrm{Bcl} 6$ expression and activate STAT3 [32, 33]. Dong et al. [15] found that the phosphorylation of STAT3 can be actively sustained after the 


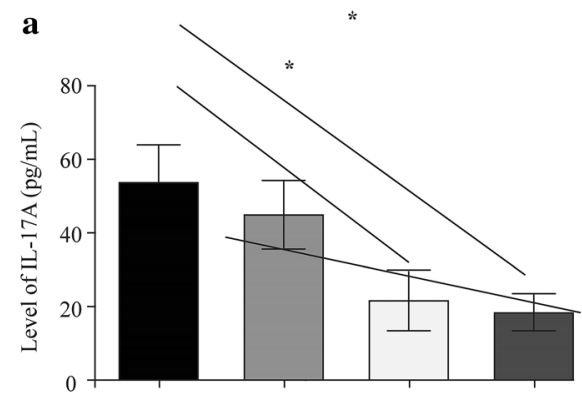

Control

$\square$ RASF+vector

$\square$ RASF+LV-miR-21

RASF+siRNA-STAT3

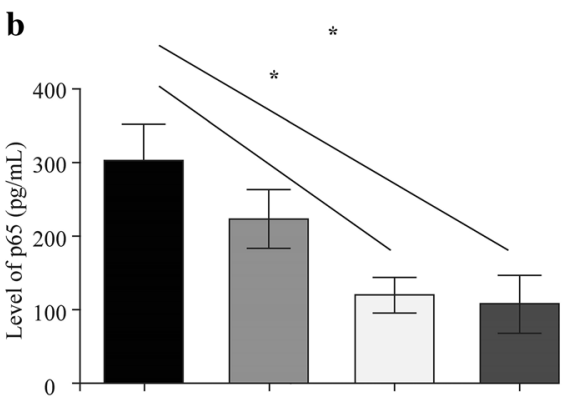

c

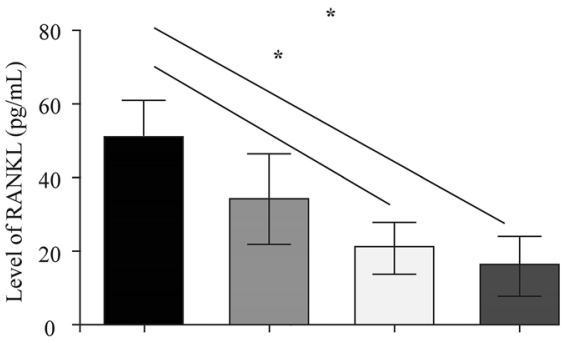

Control

$\square$ RASF+vector

$\square$ RASF+LV-miR-21

RASF+siRNA-STAT3

d

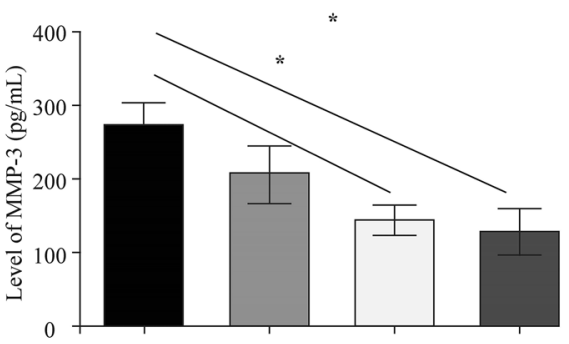

e

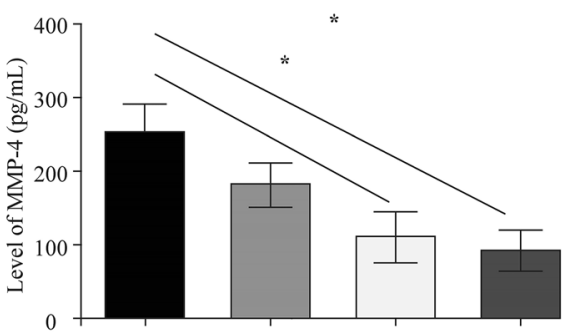

Contro

$\square$ RASF+vector

$\square$ RASF+LV-miR-21

RASF+siRNA-STAT3
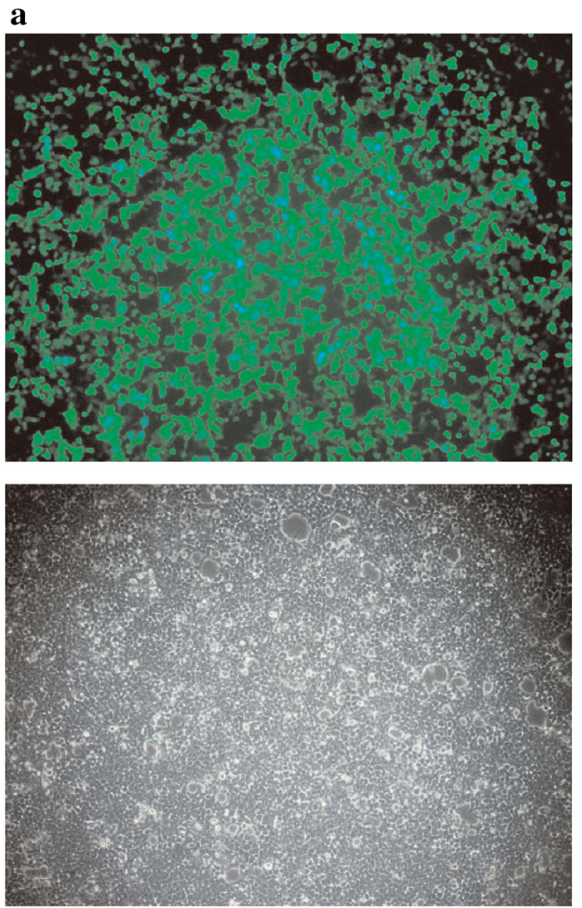

b

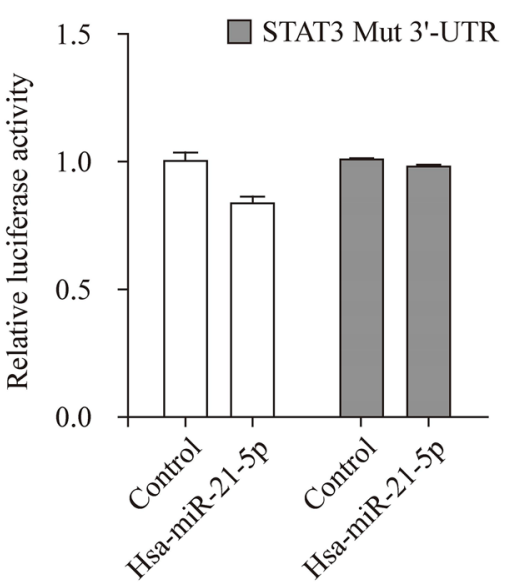

c

3. AguUguagucagac--UaudcGau Hsa-miR-21-5p

|| || || || |

5' ... ACUCUUCAgUacaUAaUAagCUU... STAT3 3'-UTR

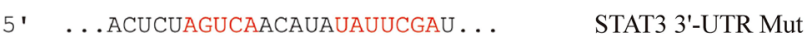

Fig. 5 The luciferase activities of the RASFs with STAT3-3'UTR dropped after being co-transfected with hsa-miR-21-5p mimics. STAT3 signal transducers and activators of transcription $3, R A S F$ rheumatoid arthritis fibroblast-like synovial cell, 3'-UTR 3'-untranslater region

knockout of miR-21 in a transplanted tumor. In tumor model, downregulation of miR-21 was associated with downregulation of STAT3 [33]. This is in contrast to data in this paper, which is upregulation of miR-21 was
Fig. 4 Enhancing miR-21 or silencing STAT3 could suppress the expression of p65 pathway. STAT3 signal transducers and activators of transcription 3, IL interleukin, $R A S F$ rheumatoid arthritis fibroblast-like synovial cell, $M M P$ matrix metalloproteinase, $R A N K L$ receptor activator of nuclear factor- $\mathrm{\kappa B}$ ligand 


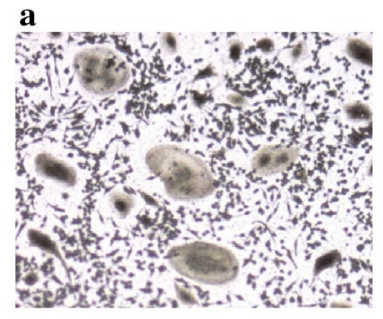

Control+M-CSF

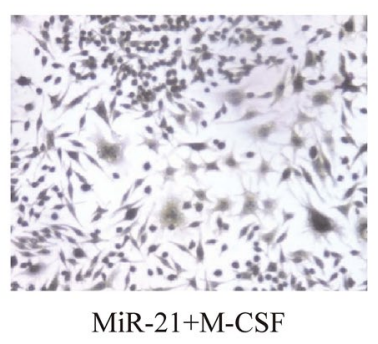

b

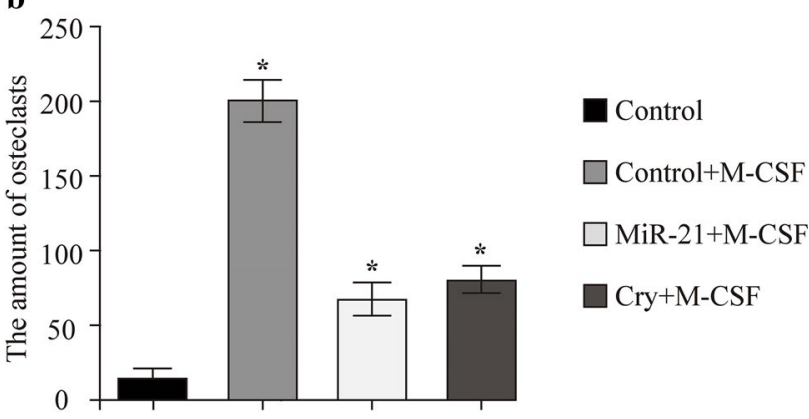

Fig. $6 \mathrm{M}$-CSF was used for inducing the RASFs to osteoclasts. RASF rheumatoid arthritis fibroblast-like synovial cell, $M-C S F$ macrophage colony-stimulating factor. $* P<0.05$

associated with downregulation of STAT3. So one will have to be very careful in drawing parallel between the malignant diseases and inflammatory diseases, and we deduce that miR-21 may inhibit production of p-STAT3the phosphorylation of STAT3. In our study, it was shown that miR-21 downregulated the expression of p-STAT3/ STAT3 and the inflammation seen in JIA is due to low miR-21. It was also identified that miR-21 could regulate the expression of some key inflammatory factors such as IL-17, p65, MMP-3, MMP-4, and RANKL by inhibiting the STAT3 phosphorylation which finally leads to the chronic inflammation and bone destruction of JIA. RANKL is a member of inflammation and bone destruction of JIA.

The decreased expression of miR-21 in JIA might be related to the differences in pathogenesis. Understanding the exact role of miR-21 in regulating JAK/STAT signal will be valuable in the development of miR-21-targeted diagnosis and therapy strategy of JIA.

Author contributions HWL contributed to the baseline collection of JIA and all the experiments. HSZ contributed to the experimental design.

Funding This study was supported by Natural Science Foundation of Guangdong Province (No. 2017A030313557), Guangzhou Health Bureau (No. 20171A011256), and Guangzhou Municipal Science and Technology Project (No. 201607010316).

\section{Compliance with ethical standards}

Ethical approval The study was performed based on an informed consent and was approved by the Ethics ethical Committee of Guangzhou Women and Children's Hospital, Guangzhou, Guangdong, China.

Conflict of interest No financial or nonfinancial benefits have been received or will be received from any party related directly or indirectly to the subject of this article.

Open Access This article is distributed under the terms of the Creative Commons Attribution 4.0 International License (http://creativeco mmons.org/licenses/by/4.0/), which permits unrestricted use, distribution, and reproduction in any medium, provided you give appropriate credit to the original author(s) and the source, provide a link to the Creative Commons license, and indicate if changes were made.

\section{References}

1. Rochette E, Duché P, Merlin E. Juvenile idiopathic arthritis and physical activity: possible inflammatory and immune modulation and tracks for interventions in young populations. Autoimmun Rev. 2015;14:726-34.

2. Singh RP, Massachi I, Manickavel S, Singh S, Rao NP, Hasan $\mathrm{S}$, et al. The role of miRNA in inflammation and autoimmunity. Autoimmun Rev. 2013;12:1160-5.

3. Roderburg C, Luedde T. Circulating microRNAs as markers of liver inflammation, fibrosis and cancer. J Hepatol. 2014;61:1434-7.

4. Filková M, Jüngel A, Gay RE, Gay S. MicroRNAs in rheumatoid arthritis: potential role in diagnosis and therapy. BioDrugs. 2012;26:131-41.

5. Kurowska-Stolarska M, Alivernini S, Ballantine LE, Asquith DL, Millar NL, Gilchrist DS, et al. MicroRNA-155 as a proinflammatory regulator in clinical and experimental arthritis. Proc Natl Acad Sci USA. 2011;108:11193-8.

6. Zhou Q, Haupt S, Kreuzer JT, Hammitzsch A, Proft F, Neumann $\mathrm{C}$, et al. Decreased expression of miR-146a and miR-155 contributes to an abnormal Treg phenotype in patients with rheumatoid arthritis. Ann Rheum Dis. 2015;74:1265-74.

7. Ma X, Wu F, Xin L, Su G, He F, Yang Y, et al. Differential plasma microRNAs expression in juvenile idiopathic arthritis. Mod Rheumatol. 2016;26:224-32.

8. Nakasa T, Miyaki S, Okubo A, Hashimoto M, Nishida K, Ochi M, et al. Expression of microRNA-146 in rheumatoid arthritis synovial tissue. Arthritis Rheum. 2008;58:1284-92.

9. Pauley KM, Satoh M, Chan AL, Bubb MR, Reeves WH, Chan EK. Upregulated miR-146a expression in peripheral blood mononuclear cells from rheumatoid arthritis patients. Arthritis Res Ther. 2008; 10:R101.

10. Kamiya Y, Kawada J, Kawano Y, Torii Y, Kawabe S, Iwata N, et al. Serum microRNAs as potential biomarkers of juvenile idiopathic arthritis. Clin Rheumatol. 2015;34:1705-12.

11. Ruan QG, Wang T, Kameswaran V, Wei Q, Johnson DS, Matschinsky F, et al. The microRNA-21-PDCD4 axis prevents type 1 diabetes by blocking pancreatic beta cell death. Proc Natl Acad Sci USA. 2011;108:12030-5.

12. Rouas R, Fayyad-Kazan H, El Zein N, Lewalle P, Rothe F, Simion A, et al. Human natural Treg microRNA signature: role of microRNA-31 and microRNA-21 in FOXP3 expression. Eur J Immunol. 2009;39:1608-18. 
13. Murugaiyan G, da Cunha AP, Ajay AK, Joller N, Garo LP, Kumaradevan S, et al. MicroRNA-21 promotes Th17 differentiation and mediates experimental autoimmune encephalomyelitis. J Clin Invest. 2015;125:1069-80.

14. Yang XO, Pappu BP, Nurieva R, Akimzhanov A, Kang HS, Chung $\mathrm{Y}$, et al. T helper 17 lineage differentiation is programmed by orphan nuclear receptors ROR alpha and ROR gamma. Immunity. 2008;28:29-39.

15. Dong L, Wang X, Tan J, Li H, Qian W, Chen J, et al. Decreased expression of microRNA-21 correlates with the imbalance of Th17 and Treg cells in patients with rheumatoid arthritis. J Cell Mol Med. 2014;18:2213-24.

16. Sun SS, Zhou X, Huang YY, Kong LP, Mei M, Guo WY, et al. Targeting STAT3/miR-21 axis inhibits epithelial-mesenchymal transition via regulating CDK5 in head and neck squamous cell carcinoma. Mol Cancer. 2015;14:213.

17. Li HW, Xie Y, Li F, Sun GC, Chen Z, Zeng HS. Effect of miR-19a and miR-21 on the JAK/STAT signaling pathway in the peripheral blood mononuclear cells of patients with systemic juvenile idiopathic arthritis. Exp Ther Med. 2016;11:2531-6.

18. ILAR 2001. Abstracts of the 20th Congress of the International League of Associations for Rheumatology. Edmonton, Alberta, Canada. August 26-30, 2001. J Rheumatol Suppl 2001;63:1-120.

19. Barnett RE, Conklin DJ, Ryan L, Keskey RC, Ramjee V, Sepulveda EA, et al. Anti-inflammatory effects of miR-21 in the macrophage response to peritonitis. J Leukoc Biol. 2016;99:361-71.

20. Sheedy FJ. Turning 21: induction of miR-21 as a key switch in the inflammatory response. Front Immunol. 2015;6:19.

21. Dong B, Shi ZH, Wang JP, Wu J, Yang ZQ, Fang KW. IL-6 inhibits the targeted modulation of PDCD4 by miR-21 in prostate cancer. PLoS One. 2015;10:e0134366.

22. Cekaite L, Clancy T, Sioud M. Increased miR-21 expression during human monocyte differentiation into DCs. Front Biosci (Elite Ed). 2010;2:818-28.

23. Landgraf P, Rusu M, Sheridan R, Sewer A, Iovino N, Aravin A, et al. A mammalian microRNA expression atlas based on small RNA library sequencing. Cell. 2007;129:1401-14.

24. Fujita S, Ito T, Mizutani T, Minoguchi S, Yamamichi N, Sakurai $\mathrm{K}$, et al. MiR-21 gene expression triggered by AP-1 is sustained through a double-negative feedback mechanism. J Mol Biol. 2008;378:492-504.

25. O'Shea JJ, Plenge R. JAK and STAT signaling molecules in immunoregulation and immune-mediated disease. Immunity. 2012;36:542-50.

26. Xie JX, Yin JH, Zhang Q, Pu R, Zhang YW, Lu WY, et al. Association of genetic polymorphisms of key molecules in JAK/STAT signaling pathway with susceptibility of hepatocellular carcinoma. Zhonghua Liu Xing Bing Xue Za Zhi. 2012;33:215-9 (in Chinese).

27. Sasi W, Sharma AK, Mokbel K. The role of suppressors of cytokine signalling in human neoplasms. Mol Biol Int. 2014;2014:630797.

28. Ju JH, Heo YJ, Cho ML, Jhun JY, Park JS, Lee SY, et al. Modulation of STAT-3 in rheumatoid synovial T cells suppresses Th17 differentiation and increases the proportion of Treg cells. Arthritis Rheum. 2012;64:3543-52.

29. Kotake S, Udagawa N, Takahashi N, Matsuzaki K, Itoh K, Ishiyama S, et al. IL-17 in synovial fluids from patients with rheumatoid arthritis is a potent stimulator of osteoclastogenesis. J Clin Invest. 1999; 103:1345-52.

30. Löffler D, Brocke-Heidrich K, Pfeifer G, Stocsits C, Hackermüller J, Kretzschmar AK, et al. Interleukin-6 dependent survival of multiple myeloma cells involves the Stat3-mediated induction of microRNA-21 through a highly conserved enhancer. Blood. 2007;110:1330-3.

31. Keul R, Heinrich PC, Müller-newen G, Muller K, Woo P. A possible role for soluble IL-6 receptor in the pathogenesis of systemic onset juvenile chronic arthritis. Cytokine. 1998;10:729-34.

32. Wang YY, Sun G, Luo H, Wang XF, Lan FM, Yue X, et al. MiR21 modulates hTERT through a STAT3-dependent manner on glioblastoma cell growth. CNS Neurosci Ther. 2012;18:722-8.

33. Zhang N, Duan WD, Leng JJ, Zhou L, Wang X, Xu YZ, et al. STAT3 regulates the migration and invasion of a stemlike subpopulation through microRNA21 and multiple targets in hepatocellular carcinoma. Oncol Rep. 2015;33:1493-8.

Publisher's Note Springer Nature remains neutral with regard to jurisdictional claims in published maps and institutional affiliations. 